ARTICLE

https://doi.org/10.1038/s41467-020-19860-0

\title{
Rapid de novo evolution of lysis genes in single-stranded RNA phages
}

Karthik R. Chamakura (1) 1, Jennifer S. Tran (1) 1,2, Chandler O'Leary (i) 1,3, Hannah G. Lisciandro (1) 1, Sophia F. Antillon (1) ', Kameron D. Garza (1) 1, Elizabeth Tran ${ }^{1,4}$, Lorna Min ${ }^{1,5}$ \& Ry Young (1) ${ }^{1 凶}$

Leviviruses are bacteriophages with small single-stranded RNA genomes consisting of 3-4 genes, one of which $(\mathrm{sg} /$ ) encodes a protein that induces the host to undergo autolysis and liberate progeny virions. Recent meta-transcriptomic studies have uncovered thousands of leviviral genomes, but most of these lack an annotated sgl, mainly due to the small size, lack of sequence similarity, and embedded nature of these genes. Here, we identify sgl genes in 244 leviviral genomes and functionally characterize them in Escherichia coli. We show that leviviruses readily evolve $\mathrm{sgl}$ genes and sometimes have more than one per genome. Moreover, these genes share little to no similarity with each other or to previously known sgl genes, thus representing a rich source for potential protein antibiotics.

\footnotetext{
${ }^{1}$ Center for Phage Technology and Texas A\&M AgriLife, Department of Biochemistry and Biophysics, Texas A\&M University, College Station, TX 778432128, USA. ${ }^{2}$ Present address: Pharmaceutical Sciences Division, University of Wisconsin-Madison, Madison, WI 53705, USA. ${ }^{3}$ Present address: University of North Texas Health Science Center, Fort Worth, TX 43210, USA. ${ }^{4}$ Present address: College of Pharmacy, University of North Texas Health Science Center,

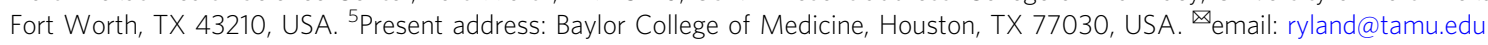




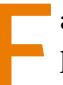
ach levivirus has three core genes, two of which encode proteins of the $T=3$ icosahedral shell: Coat (178 copies) and Mat (1 copy) $)^{1-3}$ (Supplementary Fig. 1). All known leviviruses use a retractable pilus as a receptor, recognized and bound by $\mathrm{Mat}^{4,5}$. The two best-studied leviviruses are MS2 and $\mathrm{Q} \beta$, both specific for the F conjugational pilus in E. coli. The Sgl of MS2 is L, a 75 aa product of a reading frame overlapping the end of coat and the beginning of rep $p^{6}$ (Supplementary Fig. 1). In $\mathrm{Q} \beta$, the Mat protein, called $\mathrm{A}_{2}$ for historical reasons, moonlights as the $\mathrm{Sgl}^{7}$. The lytic function of $\mathrm{A}_{2}$ derives from its direct noncompetitive inhibition of MurA, the first enzyme in the peptidoglycan (PG) biosynthesis pathway ${ }^{8}$. The lytic function of $\mathrm{L}$ is not understood mechanistically, other than it requires the chaperone DnaJ and does not involve inhibition of PG biosynthesis ${ }^{9,10}$. Until recently, only eight other Leviviridae (not counting 25 close relatives of MS2 and Q $\beta$ ) have been isolated and subjected to genomic analysis (Supplementary Table 1). These phages are specific for a wide range of retractable conjugational and motility pili $^{11,12}$. In seven of the eight cases, the $s g l$ was identified by cloning and testing in $E$. coli; ${ }^{13,14}$ no $s g l$ has been identified in the eighth ${ }^{15}$. We have recently shown that the Sgl of $\mathrm{M}$, a levivirus specific for the IncM conjugational pilus, blocks PG biosynthesis by inhibiting MurJ, the lipid II flippase ${ }^{16}$ (Supplementary Fig. 1 and Supplementary Table 1). Five of the phages have small Sgls that, although not detectably similar to MS2 L, have an L-like motif architecture that we have recently identified through genetic analysis ${ }^{17}$ (Supplementary Fig. 1). Four of these $\mathrm{sgls}$ are located roughly at the same genomic position as $\mathrm{L}$, but one, $\mathrm{L}^{\mathrm{AP} 205}$, is encoded at the extreme $5^{\prime}$ end of the gRNA $^{14}$. Taking the simplest notion, that the six L-like Sgls attack the same target (Supplementary Table 1), finding additional protein antibiotic genes has had a very limited horizon, with the sole remaining Sgl, encoded by the $C$. crescentus phage $\mathrm{Cb} 5$, already under investigation in this laboratory. However, in 2016, the tally of total ssRNA genomes deposited in the NCBI database increased by more than 10 -fold as a result of a search for ssRNA phage genomes in various metatranscriptomes ${ }^{18,19}$. Unfortunately, only one of these (AVE017) had an annotated lysis gene; in this case, the Sgl shared $\sim 38 \%$ sequence similarity to MS2 L (Supplementary Fig. 1). Even more recent studies have uncovered tens of thousands of leviviral genomes, highlighting the incredible diversity of ssRNA phages in the environment ${ }^{20,21}$.

In this work, we conducted a systematic experimental search for Sgls in the leviviral genomes. The results provide insights into not only the diversity of Sgl proteins but also into the evolution of genes.

\section{Results}

Finding candidate sgls and phenotypic analysis. Without any BLAST hits to known Sgls other than AVE017, we devised a strategy to identify the potential $s g l$ candidates in these recently discovered ssRNA phage genomes without reliance on homology searches. The first step was arbitrarily setting the minimum ORF length to 25 codons; this led to $>10$ candidates per genome, which was still too many to follow up using gene synthesis and cloning. To further winnow the possible candidates per genome, two additional criteria were introduced: a legitimate Shine-Dalgarno sequence and a predicted transmembrane domain (TMD). This narrowed the total number of candidates to $1-5$ per genome, a manageable number. We analyzed 224 genomes (complete or partial) and 158 lysis gene candidates satisfying all three criteria were identified (Supplementary Data 1). For all 158 candidates, we synthesized genes and cloned them into an arabinose inducible plasmid vector. The candidates were then tested for function in E. coli by streaking on inducing agar, scoring as positive if the colonies showed growth inhibition when compared to the empty vector control. To test the possibility of sgls falling outside of our initial criteria we also synthesized and tested an additional 135 candidates that either lacked a ribosomal binding site (RBS), fell below the 25-codon-length cutoff, or had less than an ideal TMHMM score. We found two functional genes that had no recognizable RBS and no predicted TMD (Supplementary Data 1 and Supplementary Fig. 2), five functional genes with no RBS but with a predicted TMD, three functional genes with a RBS but no predicted TMD (Supplementary Fig. 2), and one functional gene below the 25 codons in length with no RBS. All 33 candidates that scored positive on inducing agar were then tested for function by induction in liquid batch culture (Supplementary Fig. 3). There were only seven that showed overt lysis, but the other 26 showed a detectable growth inhibition phenotype; for simplicity, all of these will be referred to as functional. Considering that the natural hosts of these phages are not known, the failure to retain robust lytic function when expressed in the E. coli context is not surprising. Importantly, 178 out of 293 candidates, each with a predicted TMD, showed no effect on plating defect on inducing medium. This indicates that the simple existence of a TMD in a leviviral protein does not necessarily impose a growth inhibition or lytic phenotype under our conditions.

Mapping these $33 \mathrm{sgls}$ on their respective genomes revealed that these genes have evolved in different locations, thus creating distinct levivirus genetic architectures (Fig. 1). Almost all of the Sgls discovered in this study share no significant (>50\%) sequence similarity with each other or to any of the previously known eight Sgls from classic ssRNA phages (Supplementary Fig. 4). More strikingly, a BLAST of both the classic and new Sgls against the $\sim 16,000$ new leviviral genomes had only a few hits, thus highlighting the diversity of Sgls (Supplementary Tables 2 and 3).

A majority of functional and annotated candidate sgls are embedded in rep. To understand the genetic context of $s g l$ reading frames, each $s g l$ candidate was further binned into 18 possible genetic contexts with reference to the core leviviral genomic structure (Fig. 2a). An analysis of the genomic context of all $293 \mathrm{sgl}$ candidates revealed that most (195) were completely embedded in the two largest genes (64 in mat (region 4) and 131 in rep (region 16)). In contrast, only 15 candidates were completely embedded within the coat (region 10). Moreover, of the 33 candidates that showed activity in E. coli, more than $50 \%$ (21) are completely embedded within the rep gene in the +1 -reading frame. This apparent bias could not be explained by differences in codon usage between mat and rep genes in genomes with and without rep-embedded sgls (Supplementary Fig. 5). To better understand this bias towards evolving sgls within rep, we generated an alignment of full or near-full length Rep primary structures from the genomes with rep-embedded sgl genes (Fig. $2 \mathrm{~b}$ and Supplementary Fig. 6). The corresponding sgls (blue arrows) were then mapped onto the regions in the Rep primary structure that shared the same codon space. At first glance, this mapping revealed that rep-embedded $s g l$ genes had evolved throughout the Rep primary structure. But upon closer inspection, it is clear that most sgls are in less conserved regions of rep and can be broadly localized to two clusters; one in the N-terminal half and the other in the C-terminal half of rep. Furthermore, nearly half of the candidates in the N-terminal cluster overlap a conserved GPGA motif in Rep. This motif is conserved in the Rep primary structure and it is in the linker region connecting a pair of helices that are part of the finger domain of Rep, which has the hand overall structure $^{22}$ (Fig. 2b, c). The $s g l$ candidates that were judged to be functional and have evolved in the C-terminal half of Rep are also near a conserved motif GXFRESCG, which is part of the 


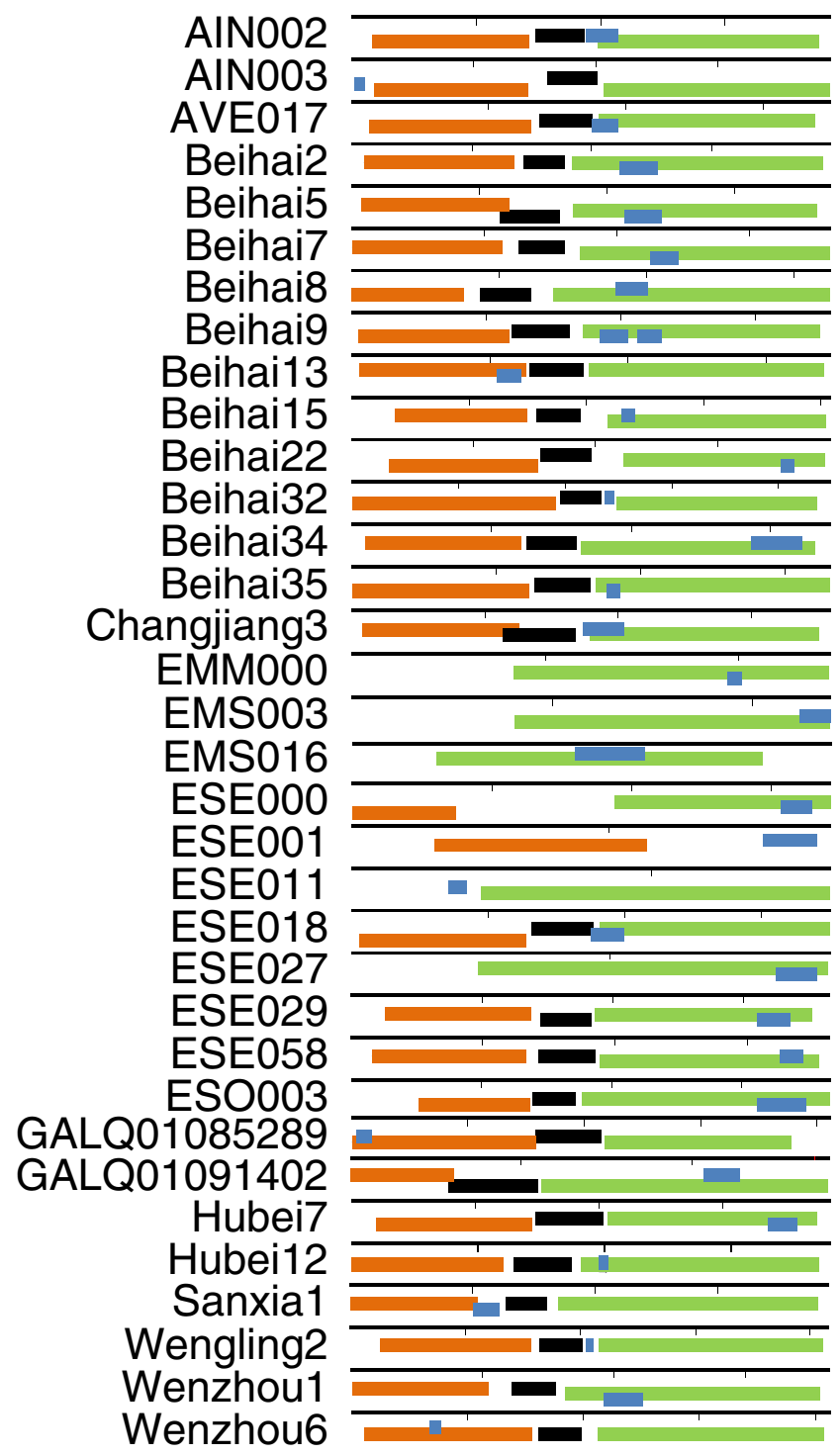

Fig. 1 Genome organization of sgls discovered in this study. The genome organization of leviviruses with functional sgls are shown and the three core genes mat, coat, and rep are colored as orange, black, and green,

respectively. The length of complete or partial genome is represented by a horizontal black bar above the genes with each perpendicular interval equal to $1 \mathrm{~kb}$. The sgls (blue) are shown relative to the reading frames of other genes (relative reading frames are represented by different horizontal levels). Beihai9 has two different sg/s in the genome and both are embedded within the rep gene.

conserved motif $\mathrm{E}$ in $\mathrm{RdRps}^{23}$. Mutations in the first Gly in GXFRESCG motif are lethal to the function of replicase, which points to the high degree of constraints on base composition in the corresponding regions of the RNA. Only two of the $s g l s$ that showed function in E. coli (GLAQ01091402 and EMM000) have evolved within the highly conserved central region (motifs A-E) of Rep. To gain insights into the effect $\mathrm{sgl}$ reading frames have on the tertiary structure of Rep, we mapped the regions that gave rise to Sgls onto the corresponding amino acids in the crystal structure of Q $\beta$ Rep. Strikingly, almost all regions of the structure have evolved Sgls, including the core catalytic regions (Fig. 2c). We wondered if this broad clustering of sgls within the Rep primary structure was also observed for the candidates that scored negative in our initial screen in E. coli. We aligned the respective Rep sequences and then mapped the failed $s g l$ candidates on the alignment (Fig. 2d). Surprisingly, most of the candidates still mapped to the less conserved $\mathrm{N}$ - and C-termini of Rep. Only a few of them are fully embedded in the central highly conserved region.

ssRNA phage genomes with more than one sgl per genome. Of the $293 \mathrm{sgl}$ candidates cloned and tested from 244 partial or nearcomplete genomes, only 168 genomes had sgl candidates, with some having up to five candidates per genome (Fig. 3 and Supplementary Data 1). Eighty-nine genomes had two candidates, 25 had three candidates, 10 had four candidates, and one had five candidates. However, none of them had two sgls that exhibited function in E. coli. We reasoned that some ssRNA phages in their natural environments might be capable of infecting two or more evolutionarily distant hosts and thus might require multiple sgls. For example, phage PRR1 infects both E. coli and Pseudomonas carrying the RR1 multiple-drug resistance plasmid (Supplementary Table 1). To test this hypothesis, we focused on 20 genomes that had at least two sgl-candidates within the rep gene. The Beihai9 genome had two candidates, with only one candidate (Beihai9_1) exhibiting function in E. coli in our initial screen (Fig. 4a). We sought to evolve Beihai9_2 through directedevolution to gain lytic activity in $E$. coli. We constructed a PCRmutagenized Beihair9_2 plasmid library consisting of 5000 clones and used the plasmid release technique ${ }^{10}$ to enrich for mutant clones that now caused lysis. After two rounds of enrichment, 20 clones were tested, revealing two clones (123 and 131) that caused growth inhibition on inducer plates; both caused lysis after induction in liquid culture (Fig. 4b). Sequencing the two clones revealed that there were multiple mutations in both, with clones 123 and 131 having 3 and 5 single base changes, respectively (Fig. 4c). Reconstructing all eight of the single base changes separately in the parental plasmid did not restore the lytic phenotype. However, one set of double mutants (g15t and A52D) restored the lysis phenotype (Fig. 4d). Interestingly, the double mutant consisted of a silent $\mathrm{G} \rightarrow \mathrm{T}$ base change, which suggests that RNA structure might play a role in expression of the Sgl from the plasmid. Nevertheless, the results show that leviviruses are capable of carrying more than one lysis gene or an easily adaptable cryptic gene in addition to the main $\mathrm{sgl}$.

Evolution of Sgls in closely related ssRNA phages. To gain a better understanding of the evolution of $\mathrm{sgls}$ in closely related ssRNA phages, we constructed cladograms based on the full or near-full length Rep or Mat sequences (Fig. 5a and Supplementary Fig. 7). Only the nodes with bootstrap values $>80$ were further analyzed. Based on this cutoff, 133 of the 174 Rep sequences considered could not be grouped into clades but the remaining 41 Rep sequences could be grouped into 8 clades. Of the clades that could be formed, the largest had 12 members and the smallest had three (Fig. 5a). In Fig. 5a, we highlighted the phage names with either blue (Sgls discovered in this study) or black (classic Sgls from Supplementary Table 1). To analyze the evolution of $s g l s$ within closely related phages, we restricted our analyses mainly to nodes with bootstrap values $>98$, but exceptions were made if one of the phages had a functional Sgl (blue or black highlight). A total of 44 Rep sequences fit the above criteria and their corresponding genomes were compared as multiple sequence dot plots to find similarity across the length of the genome (Supplementary Fig. 8). These analyses showed that 11 of the 44 genomes have varying degrees of nucleotide similarity across the genome (Fig. 5b). Most strikingly, at the nucleotide level the regions corresponding to the mat gene ( $5^{\prime}$-half of the genome) were more divergent than the $3^{\prime}$-half of the genome, which suggests that changes in host-specificity determinant (Mat) 
a

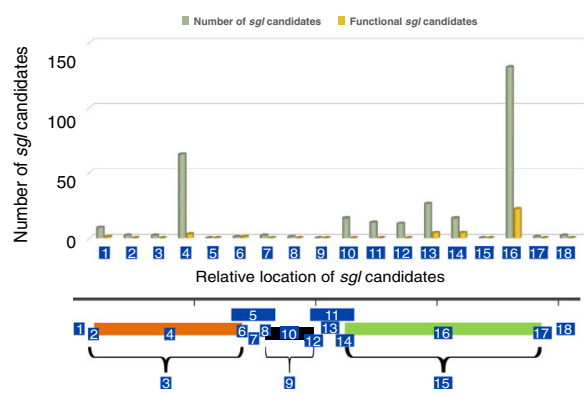

b

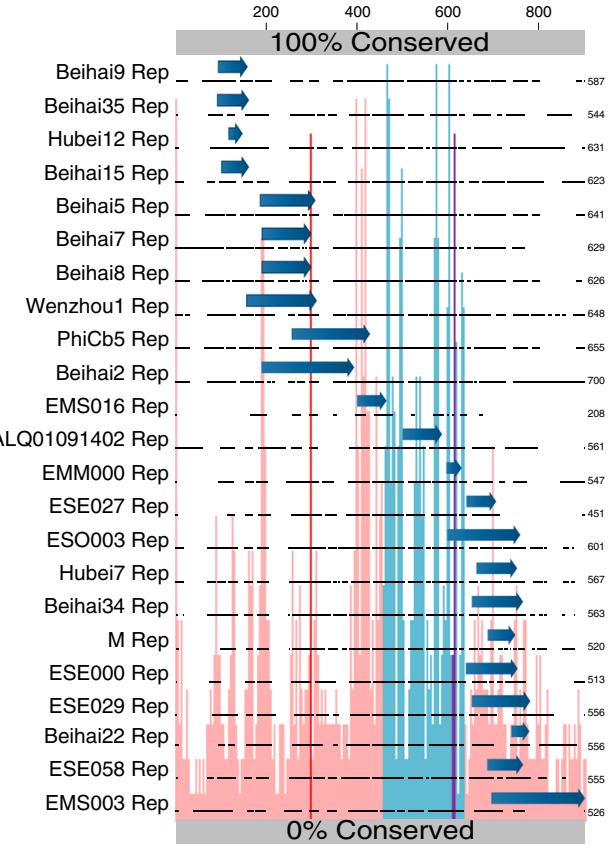

C

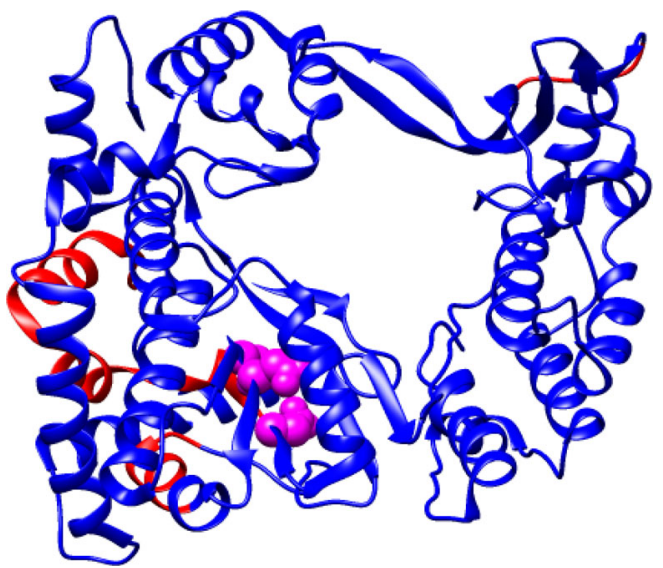

d



Fig. 2 Genomic hot spots for sgl evolution. a Relative location of sgl-candidates within a canonical ssRNA phage genome architecture. Numbers 1 through 18 refer to the sub-locations within the genome where sgl-candidates could potentially be found. The bars in the graph show the total number of sglcandidates (light green) and functional sgls (orange) per sub-location. b An alignment of Rep sequences from phage genomes that have rep-embedded functional sgls. The regions of sequence similarity are shown as lines and the gaps are shown as breaks in the lines. The location of the $s g /$ in the context of replicase primary structure is shown as a blue arrow above the line. Sequence conservation among the Rep sequences is shown as pink bars ( $0 \%$ at the bottom gray bar, $100 \%$ conservation top gray bar). The bars corresponding to conserved motifs are colored as follows GPGA $=$ red; $m$ otifs $A-E=$ light blue; FRESCG = purple. $\mathbf{c}$ The relative location of functional sgls mapped on to the crystal structure of Q $\beta$ RNA-Dependent RNA polymerase (RdRp) beta subunit (PDB:4R71). The structural elements that have tolerated evolution of embedded sgls are highlighted in blue. The catalytically important residues are shown as magenta spheres and the regions without any functional sg/s are highlighted red. $\mathbf{d}$ An alignment of Rep sequences from phage genomes that have rep-embedded non-functional sgl candidates with $\mathrm{Q} \beta$ Rep as a reference at the top. Annotations are the same as in $\mathbf{b}$, except the secondary structure elements (alpha helices $=$ green rectangles; beta strands = orange rectangles) are annotated above the $\mathrm{Q} \beta$ Rep. 


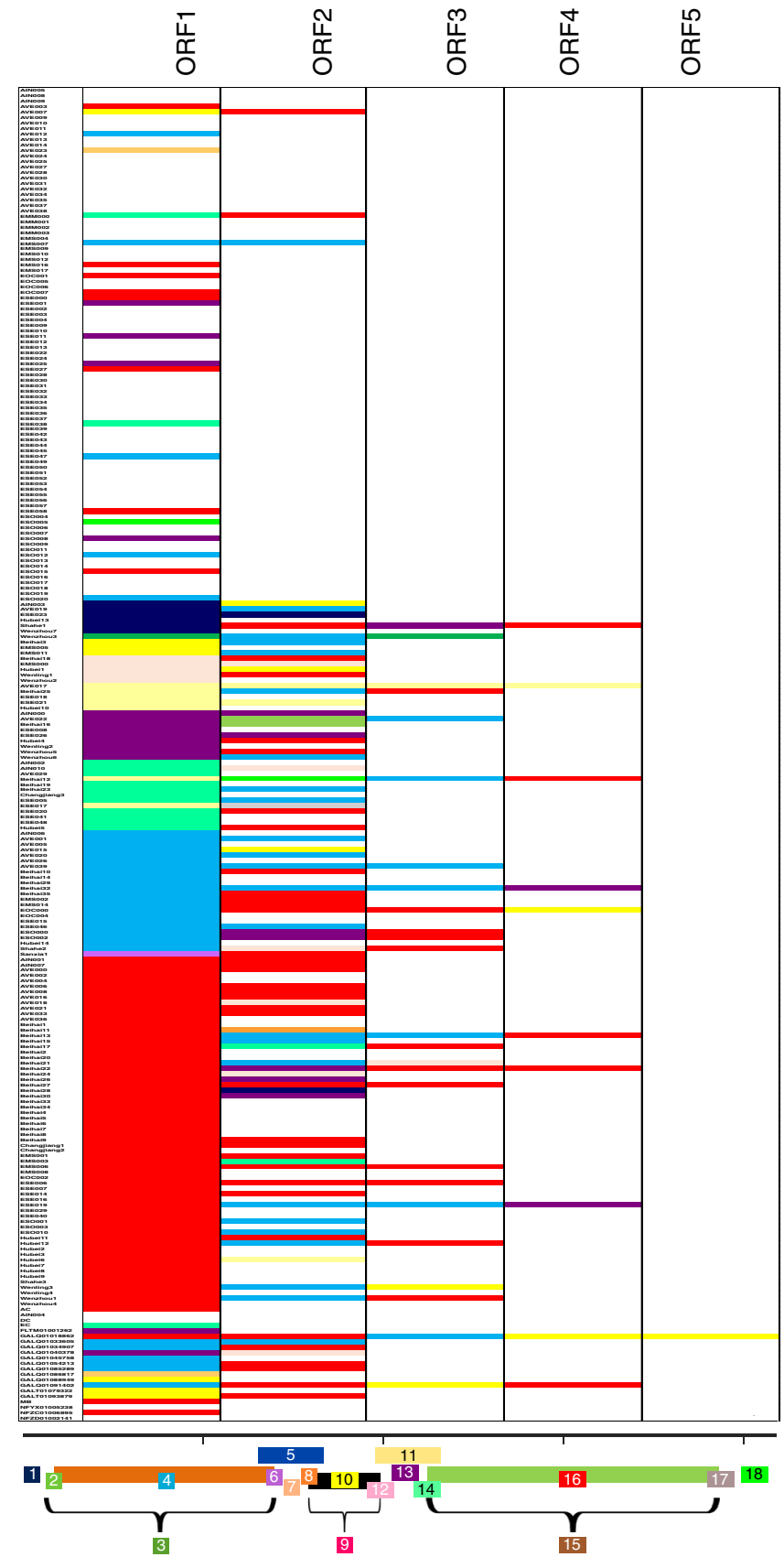

Fig. 3 Relative location of sgl-candidate ORFs. The number and relative location of sgl-candidate ORFs that were synthesized and tested per genome. The relative genomic locations (1-18) are shown on the canonical ssRNA phage genome and color coded as follows: 1 (navy blue), 2 (lime green), 3 (army green), 4 (teal blue), 5 (royal blue), 6 (orchid purple), 7 (tan), 8 (orange), 9 (bright pink), 10 (bright yellow), 11 (pale yellow), 12 (rose pink), 13 (dark purple), 14 (seafoam green), 15 (brown), 16 (red), 17 (gray), 18 (neon green).

occur more frequently than in either coat or rep. We wondered if changes in the mat gene correlated with the changes in the corresponding sgl. Based on the genomic context of the sgls, the 11 pairs could be separated into two sub-classes; the D-class (different) consisting of genome pairs where the $s g l$ or $s g l$-candidates arose in different genomic locations and the S-class (similar) where the genetic context remained the same but the sequence has diverged (Fig. $5 b$ ). Eight genome pairs belonged to the Dclass, of which two pairs (Sanxia1/Wenzhoul and Wengling2/
Wenzhou6) had functional sgls that had evolved in different locations within the respective genomes. Of the remaining six pairs, only one of the phages in each pair had a functional $s g l$ while the corresponding sequence in the other phage diverged away, as a new sgl evolved in a different location. The evidence for such divergence is found in the sequence of the corresponding phage pairs. In the case of the phage M/Beihai25 pair, $l y s^{M}$ the $s g l$ of phage $\mathrm{M}$ is embedded in the $3^{\prime}$-half of rep gene, but the corresponding region in Beihai25 lacks an ORF. Nevertheless, the remnants of the $l y s^{M}$ gene can still be found in the Beihai25 genome (Fig. 5b and Supplementary Fig. 9). Similar observations of gene loss can be made in Beihai15/Beihail6. Among the three S-class phage pairs, only MS2/AVE017 have $s g l s$ that tested functional; even here, the Sgls share only $38 \%$ amino acid sequence identity. Interestingly, the S-class pair Hubei7/Hubei8 have $s g l s$ that failed the initial function test in E. coli. The putative Sgls share $\sim 47 \%$ sequence identity, with the predicted TMDs differing at eight positions and with highly divergent periplasmic domains (Fig. 5c). Taken together, the conservation of the elements that make up a gene, such as Shine-Dalgarno and start/ stop codons, similar gene length, conservation of predicted TMDs, and sequence identity over the length of the protein suggests that these ORFs are functionally relevant in the native hosts. To test this hypothesis, we used the plasmid release method

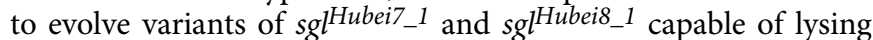
E. coli (Fig. 5c). Of the $24 s g l^{H u b e i 7_{-} I}$ clones screened after two rounds of plasmid release, six unique mutants were isolated. All six unique gain-of-function mutants of $s g l^{H u b e i 7_{1} 1}$ have at least one mutation localized around Trp15, with three mutants replacing Trp with Leu, while the other three mutants have missense changes at position 14 or 16 (Fig. 5c). Phenotypically, the three single missense mutants (mut5 (L16I), mut6 (W15L), and mut15 (L16F)) display two different lysis profiles, with the former two causing a rapid drop in optical density and the latter displaying a more gradual drop (Fig. 5d). On the other hand, mutations in the gain-of-function mutants of $s g l^{H u b e i 8_{-} 1}$ are predominantly localized in the highly divergent predicted periplasmic domain (Fig. 5c, e). In addition, a missense change near the N-terminus (S3F) also appears to be sufficient to elicit lysis in E. coli (Fig. 5c). Thus, it is clear from the above experiments that both $s g l^{H u b e i 7}{ }_{1}$ and $s g l^{H u b e i B_{1}}{ }^{1}$ are functionally relevant $s g l s$ that have diverged away from each other, presumably after the phage adapted to infect a different host species. Moreover, this result suggests that many, if not most, of our candidate Sgls that failed the function test in E. coli are likely to be real Sgls in the context of the most common host.

De novo gene evolution in genomes sourced from geographically proximal regions. The disparate $s g l s$ discovered in leviviruses sourced from global metatranscriptomics suggests that some arose through de novo gene evolution events. To understand such events in the context of both the geographical location and the source of the metatranscriptomes, we focused on the genome pairs Sanxia1/Wenzhoul and Wengling2/Wenzhou6 which show extensive sequence similarity but have evolved functional $s g l$ genes in different places (Fig. 5b). These four genomes were discovered from the metatranscriptomes of invertebrate animals sourced from regions within a $\sim 200 \mathrm{~km}$ radius near the East China Sea (Fig. 6a). Sanxial was sourced from a shrimp on the island of Taiwan, while Wenzhoul/ Wenzhou6 and Wenling2 came from an apple snail from the city of Wenzhou and crustacean mix from the city of Wenling, respectively. The dot plot of the genomes Sanxial and Wenzhoul shows overall sequence similarity in the first $~ 3000$ bases with only six distinct regions (boxed and numbered 1-6) 
a

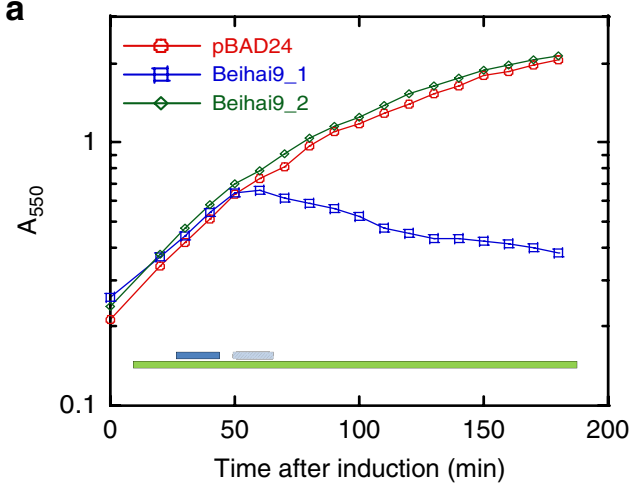

b

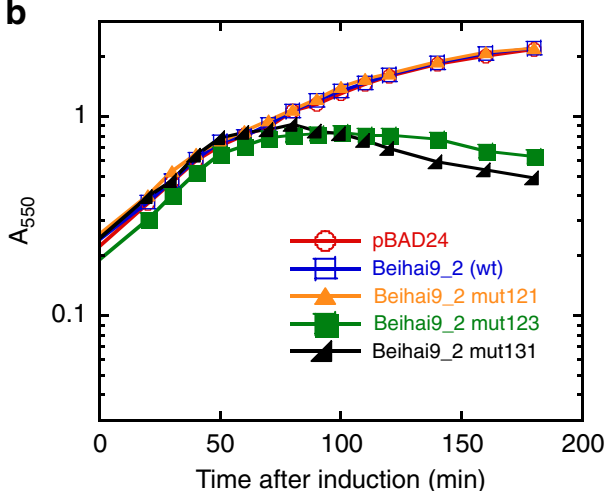

C

\begin{tabular}{|c|c|c|}
\hline Mutant & $\begin{array}{c}\text { Base } \\
\text { changes }\end{array}$ & $\begin{array}{c}\text { Amino acid } \\
\text { changes }\end{array}$ \\
\hline \multirow{4}{*}{123} & G15T & No change \\
\cline { 2 - 3 } & C161A & A54D \\
\cline { 2 - 3 } & T174C & No change \\
\hline \multirow{4}{*}{131} & G15T & No change \\
\cline { 2 - 3 } & C28T & L10F \\
\cline { 2 - 3 } & T56C & L19F \\
\cline { 2 - 3 } & A62T & Y21F \\
\cline { 2 - 3 } & T151G & L51V \\
\hline
\end{tabular}

d

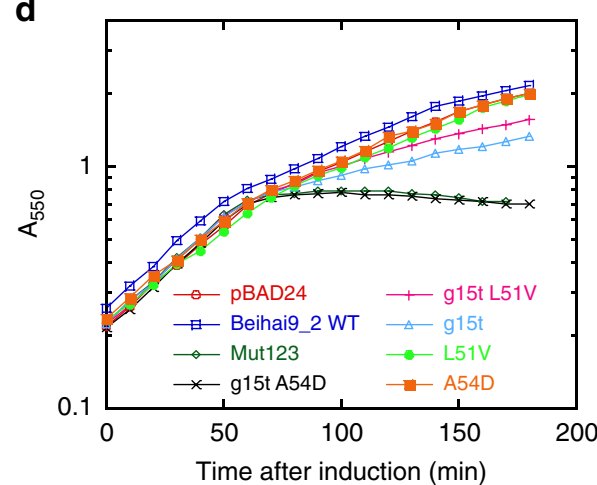

Fig. 4 ssRNA phage with two functional lysis genes. a Lysis profiles of two sgl candidates (Beihai9_1 and Beihai9_2); pBAD24 (red open circle), Beihai9_1 (blue open squares), Beihai9_2 (green open diamond).The genomic context of sgls is shown relative to the rep gene (green bar) with the functional candidate (Beihai9_1) shown as dark blue and the non-functional candidate as light blue. $\mathbf{b}$ The lysis profiles of Beihai9_2 gain-of-function mutants 123 and 131; pBAD24 (red open circle), Beihai9_2 (blue open square), Beihai9_2 mut121 (light orange-filled triangle), Beihai9_2 mut123 (green-filled square), Beihai9_2 mut131 (black-filled right triangle). c Table summarizing the base and amino acid changes of the mutants in $\mathbf{b}$. d The lysis profiles of Beihai9_2 of single and double mutant constructs; pBAD24 (red open circle), Beihai9_2 (blue open squares), Beihai9_2 mut123 (green open diamond), Beihai9_2 double mutant g15t A54D (black cross), Beihai9_2 double mutant g15t L51V (pink plus), Beihai9_2 g15t (light blue open triangle), Beihai9_2 L51V (light green-filled circle), and Beihai9_2 A54D (orange-filled squares). The lysis profiles shown here are representative of three biological replicates.

lacking similarity (Fig. 6b). Mapping of the respective genetic architectures on the dot plot revealed that sgls in Sanxial and Wenzhoul arose in regions 4 and 6 , respectively. Of the remaining four boxed regions, boxes 1, 2, and 3 encompass the region encoding the Mat protein, which provides pilin-specificity and attachment to the host pilus. A protein dot plot of the Mat proteins from Sanxial and Wenzhoul showed that the $\mathrm{N}$ terminal half or the putative pilin binding half of the proteins diverged from each other (Fig. 6c). Similarly, the sgls in other two highly similar phages, Wenling2 and Wenzhou6, arose in places that diverged away from each other and the genomes also differ in the regions encoding $\mathrm{N}$-terminal half of Mat proteins (Fig. $6 \mathrm{~d}$ and Supplementary Fig. 10). Interestingly, the observation that genome differences also map to mat genes suggests that changes in the Mat protein alter the pili-specificity and confer the ability to infect a different and possibly distant host species. This adaptation to evolutionarily distant hosts likely renders the existing $s g l$ useless and drives the evolutionary pressure to evolve a sgl de novo (Fig. 6e). To understand the specific base changes that led to the birth of a sgl gene in Sanxial, we compared the nucleotide sequence of sgl Sanxial (bases 968..1228) to the corresponding region in Wenzhoul (bases 975..1183). The alignment showed that $\mathrm{sgl}^{\text {Sanxial }}$ arose de novo from a stretch of bases without any recognizable ORF in any of the three reading frames (Fig. 6f). Interestingly, one of the reading frames has 11 aa that are conserved in Sgl ${ }^{\text {Sanxial; }}$; moreover, to give rise to a $s g l$, the reading frame underwent 45 single base changes and 14 separate indels ranging in size from 1 to $12 \mathrm{bp}$ (Fig. $6 \mathrm{f}$ ).

\section{Discussion}

Recent advances in next-generation sequencing technologies and directed searches for RNA viruses have either intentionally or unintentionally facilitated the discovery of thousands of leviviruses $^{18-21}$. However, almost all of the ssRNA phage genomes sourced from metatranscriptomes lack an annotated lysis gene or $s g l$, which is not surprising because most of the known Sgls are small, are encoded in alternate reading frames of essential genes, and lack sequence similarity to other Sgls. To tackle the missing gene problem, we took the simple approach of identifying ORFs in 244 genomes, cloning the putative $s g l$ candidates on inducible plasmids, and testing them for lytic or growth inhibitory effect in E. coli. This led to the identification of 35 unique Sgls (33 in the initial screen plus two evolved Sgls) exhibiting activity in E. coli, each potentially representing a distinct mechanism to effect host cell lysis. Moreover, a BLAST search with the expanded pool of Sgls against the recently deposited tens of thousands of leviviruses returned only a handful of hits, which suggests that Sgls are extremely diverse and remain vastly untapped as a source for peptides that attack essential cellular functions; i.e., for protein antibiotics $^{24,25}$.

One lysis gene per phage genome has been the paradigm for small lytic phages (microviruses and leviviruses) since the late 


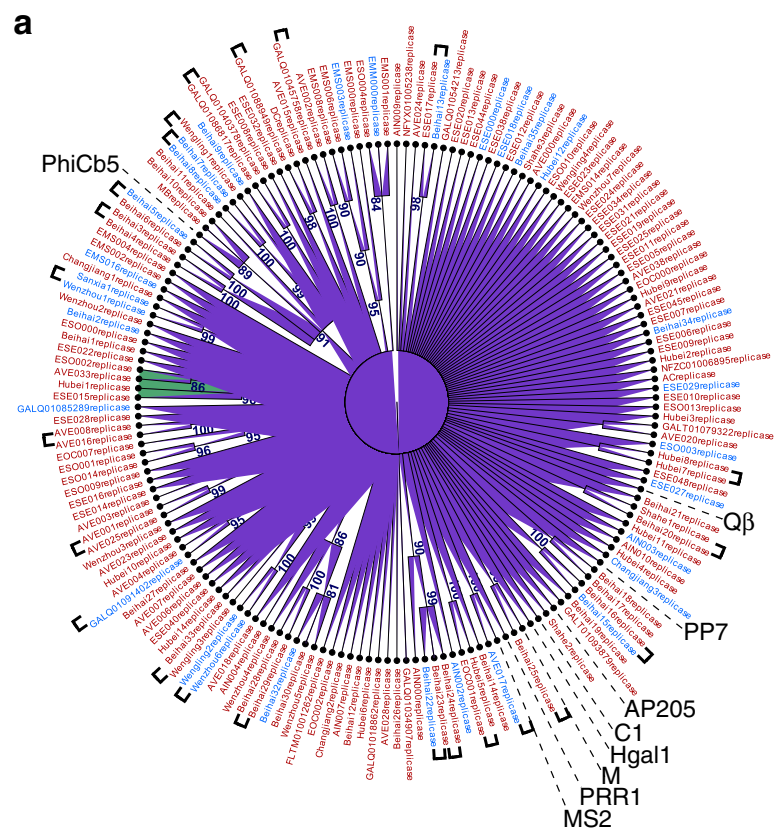

b

C

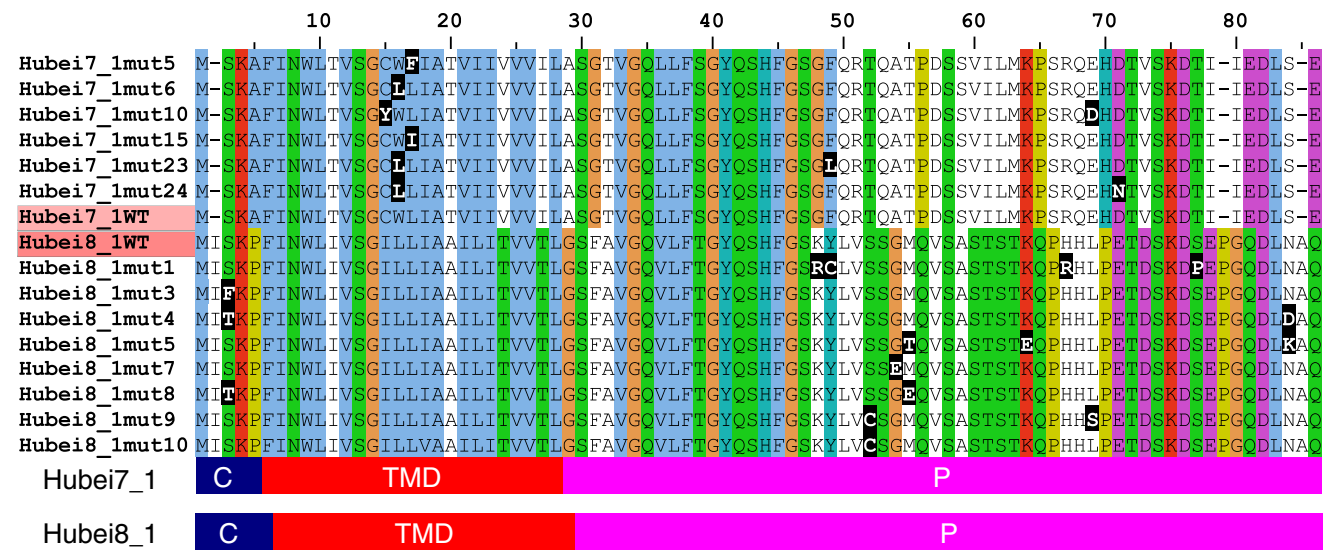

d

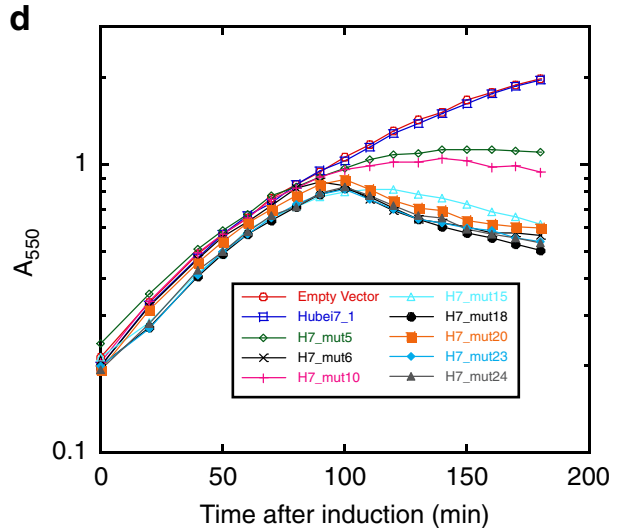

1970s, when the first genomes, $\varphi$ X174 and MS2, respectively, were sequenced 26,27 . The results presented in this study show that a ssRNA phage could potentially have two or more lysis genes at different stages of gene evolution. One reasonable hypothesis for multiple $s g l s$ per genome is that leviviruses are capable of infecting different host species, mainly due to their retractable pilus tropism, rather than specificity to host surface receptors. Therefore, the selective pressures to maintain more than one $s g l$ per genome may arise in an environment of near constant
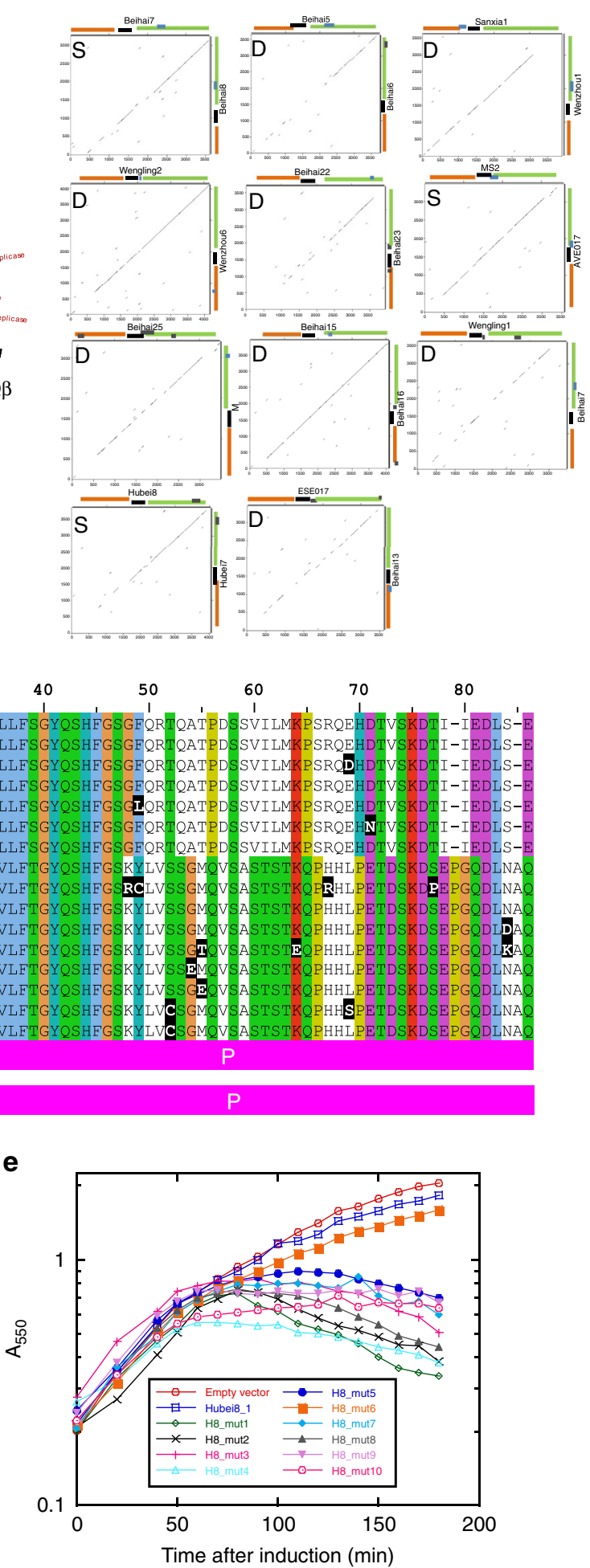

passage of the virus from one species to the other. In the absence or loss of such pressure, the other sgls diverge in their sequence to become cryptic and ultimately lose the basic features of a gene. The evolution of disparate $\mathrm{sgls}$ in closely related phages suggests rapid gene evolution, but the timescale and frequency of such events is not known. By mining the ssRNA phage sequence space and doing in vitro reconstruction experiments, insights into such events and the evolutionary steps that turn a stretch of nucleic acids into a new gene with a new function could be obtained. 
Fig. 5 Evolution of Sgls in closely related ssRNA phages. a Cladogram of ssRNA phages based on the corresponding Rep primary structures. Nodes with bootstrap values $>80$ (resampling $n=1000$ ) are shown and nodes with at least two levels are grouped as clades and are highlighted in eight different colors. The genomes with sgls exhibiting function are colored blue, the sgls from the previously characterized ssRNA phages are colored black, and candidates that tested as non-functional are colored red. Closely related phages are indicated by brackets. $\mathbf{b}$ Nucleotide dot plots of full length contigs of the closely related ssRNA phage genomes ( $n=11$ genome pairs). Each dot plot compares two genomes and the dot plots are categorized as $S$ (similar) and D (different) based on the relative genomic context of sgls. c An alignment of Hubei7_1 and Hubei8_1 wild-type and gain-of-function mutants. The amino acids in the alignment are highlighted according to clustalX and the missense changes found in gain-of-function mutants are highlighted as white letters on black background. The respective predicted membrane topologies are shown below the alignment as bar diagrams with different colors representing different predicted subcellular localizations. Blue (cytoplasmic, C), red (transmembrane domain, TMD), and purple (periplasmic, P). d The lysis profiles of gain-of-function Hubei7 mutants; pBAD24 (red open circle), Hubei7_1 (blue open squares), H7_mut5 (green open diamond), H7_mut6 (black cross), H7_mut10 (pink plus), H7_mut15 (light blue open triangle). H7_mut18 (black-filled circle), H7_mut2O (orange-filled square), H7_mut23 (blue-filled diamond), H7_mut24 (light gray-filled triangle). The mutants H7_mut6, mut18, and mut20 have the same change (W15L). e The lysis profiles of gain-offunction Hubei8 mutants; pBAD24 (red open circle), Hubei8_1 (blue open squares), H8_mut1 (green open diamond), H8_mut2 (black cross), H8_mut3 (pink plus), H8_mut4 (light blue open triangle). H8_mut5 (blue-filled circle), H8_mut6 (orange-filled square), H8_mut7 (blue-filled diamond), H8_mut8 (light gray-filled triangle), H8_mut9 (light pink-filled inverted triangle), H8_mut10 (pink circled dot). H8_mut1 and mut2 are siblings and H8_mut6 (S53P and S58P) is non-functional. The lysis profiles shown in this figure are representative of three biological replicates.

Another wide-ranging impact of this study comes from the observation that a large proportion of $\mathrm{sgls}$ have evolved completely within the rep gene, specifically in the +1 reading frame. Even though mat and rep genes are similarly sized, a disproportionate number (22 of 35) of sgls or sgl-candidates were found embedded within the rep gene. This disparity could not be explained by differences in sequence attributes such as codon usage. Interestingly, most of the shared sequence space in the rep gene encodes highly divergent regions of Rep, with the near universally conserved catalytic regions of Rep (motifs A and C) harboring only a few sgls. Therefore, it is reasonable to hypothesize that the highly divergent regions are more permissible for exploration of codon-space, especially in alternate reading frames, and thus serve as hotspots for $s g l$ evolution. Moreover, Rep relies on host proteins such as ribosomal protein S1, EF-Tu, EF-Ts, and $\mathrm{Hfq}$ (for $\mathrm{Q} \beta$ ) to form a replication-competent holoenzyme ${ }^{28-31}$. These host proteins could also serve as a structural scaffold to attain the optimal conformation of RdRp. Thus, this added flexibility allows the rep gene to explore sequence space much more than mat and coat. Evolutionarily, the leviviruses are thought to be ancestors of capsid-less eukaryotic viruses such as mitoviruses and narnaviruses, which are found in fungi as naked RNA replicons in the mitochondria and the cytosol, respectively $^{32}$. It has been speculated that RdRp genes were passed on through modular gene exchange to various lineages of eukaryotic RNA viruses. Hence, it might be worthwhile to investigate the RdRp genes from other RNA viruses for the propensity to carry small embedded genes that may play a critical role in pathogenesis.

By analyzing a relatively minuscule sample of the total leviviral universe or leviverse, we have uncovered an incredible diversity of small peptides that carry out a critical function in the life cycle of RNA viruses. Our results motivate further research into exploiting these peptides for identifying targets for antibiotic development, to uncover small genes and their biological functions in RNA viruses of higher eukaryotes, and provide a good model system for studying de novo gene evolution and origins.

\footnotetext{
Methods

Bacterial strains, plasmids, primers, and growth conditions. The plasmids used in this study are listed in Supplementary Data 1 and Supplementary Table 4. Bacterial strains used in this work are XL1Blue (Stratagene) and DH5a (ThermoFisher Scientific). Primers and synthetic DNA (g-blocks) are listed in Supplementary Data 2. Cultures were grown with aeration at $37^{\circ} \mathrm{C}$ in lysogeny broth (LB) supplemented with ampicillin $\left(100 \mu \mathrm{g} \mathrm{mL}^{-1}\right)$ and L-arabinose $(0.4 \% \mathrm{w} / \mathrm{v})$ when indicated.

The $s g l$ candidate ORFs (with or without codon optimization for E. coli) flanked by restriction sites for EcoRI and XhoI/HindIII upstream and downstream,
}

respectively, were synthesized as g-blocks (IDT). Each g-block contained two to five $s g l$ candidates with each candidate flanked by the above restriction sites to facilitate cloning. The g-blocks were digested with EcoRI/HindIII or EcoRI/XhoI enzymes and cloned into similarly digested pBAD24 (empty vector) or pKC3, respectively ${ }^{16}$. The clones were verified by Sanger sequencing (EtonBiosciences) with primers KC30 or KC31.

Mutant alleles of $s g l^{\text {Beihai9_2 }}$ were constructed by site-directed mutagenesis on the plasmid pBAD24 Beihai9_2 using Phusion high-fidelity DNA polymerase and the primers KC737, KC738, KC739, KC740, KC741, KC742, KC745, KC746, KC747, KC748, KC749, KC750, KC751, KC752, KC753, KC754, KC755, and KC756.

Chemical reagents and enzymes. All chemicals were purchased from SigmaAldrich unless otherwise stated. All enzymes and the associated buffers were purchased from New England Biolabs unless otherwise stated.

Annotation of sgl-candidates. The ssRNA phage genomes were sourced from previously deposited and/or published datasets ${ }^{18,19}$; see Data Availability, below. The $s g l$ candidates in these genomes were manually annotated using SnapGene (GSL Biotech LLC). All ORFs greater than 25 amino acids with either ATG or GTG or TTG start codons were selected, analyzed for a putative Shine-Dalgarno sequence, and the corresponding protein sequences were analyzed for predicted transmembrane domain (TMD) using TMHMM server, v.2.0 http://www.cbs.dtu. $\mathrm{dk} /$ services/TMHMM/. The ORFs that passed the above three criteria were synthesized, cloned, and assayed for lytic function in E. coli XL1Blue. One or more exceptions to the three criteria listed above were made while annotating some $s g l$ candidates.

Functional characterization of sgl-candidates. The transformants carrying the $s g l$ plasmids were streaked on inducer $(0.4 \% \mathrm{w} / \mathrm{v} \mathrm{L}$-arabinose $)$ and non-inducer LB agar plates supplemented with ampicillin $(100 \mu \mathrm{g} \mathrm{mL}-1)$ and incubated at $37{ }^{\circ} \mathrm{C}$ overnight. The clones that showed growth inhibition only on the inducer plates were scored as positive hits. The positive clones were further characterized by following their lysis profiles. Lysis profiles were obtained by taking $125 \mu \mathrm{L}$ of overnight cultures and adding them into respective $250 \mathrm{~mL}$ culture flasks with $25 \mathrm{~mL}$ of LB supplemented with ampicillin $(100 \mu \mathrm{g} / \mathrm{mL})$. The flasks were incubated in a $37{ }^{\circ} \mathrm{C}$ water bath shaker and induced at $\mathrm{A}_{550}=0.2$ with arabinose. After induction, the optical density was determined at regular intervals and the data was plotted using Kaleidagraph version 4.03 (Synergy Software).

Mutant library construction and plasmid release. The lysis gene candidates were mutagenized using the GeneMorph II Random Mutagenesis Kit (Agilent Technologies) as per manufacturer's instructions using primers KC30 and KC31. To ensure high mutation rate, low initial template $(1 \mathrm{ng} / \mu \mathrm{L})$ and 35 cycles of amplification were used. The PCR product was digested with DpnI to remove template plasmid DNA and purified using PCR clean up kit (Qiagen). The purified mutant PCR product was digested with EcoRI and HindIII and ligated into similarly cut pBAD24 vector. The ligated product was then transformed into MAX Efficiency DH5a-T $1^{\mathrm{R}}$ competent cells (ThermoFisher Scientific). The mutation frequency was assessed by sequencing 10 colonies and the cloning process was repeated until desired number of colonies were obtained.

To pool the mutant library, $5 \mathrm{ml}$ of LB was added per plate and the colonies were scraped off the plate using a sterile glass rod. The pooled cell suspension was diluted 10 -fold and $125 \mu \mathrm{L}$ of the diluted suspension was used to inoculate $25 \mathrm{~mL}$ LB media supplemented with ampicillin $(100 \mu \mathrm{g} / \mathrm{mL})$ in a $250 \mathrm{~mL}$ Erlenmeyer flask. Cultures were aerated at $37^{\circ} \mathrm{C}$ in a water bath shaker (New Brunswick Scientific 
a

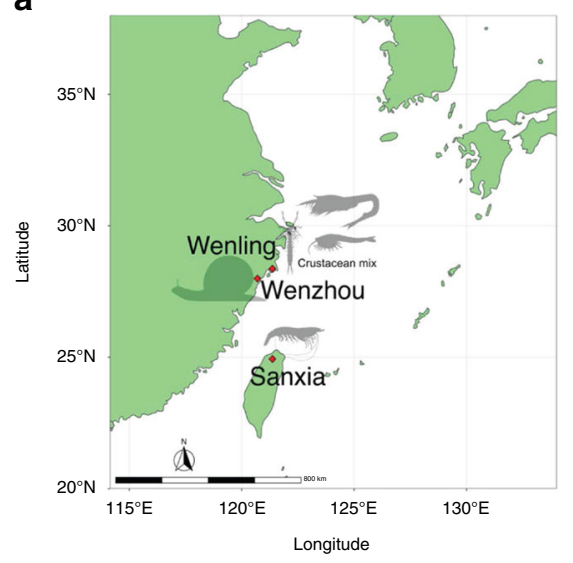

C

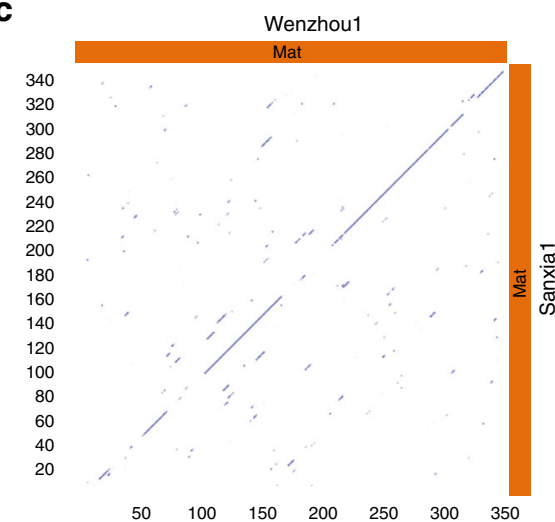

b

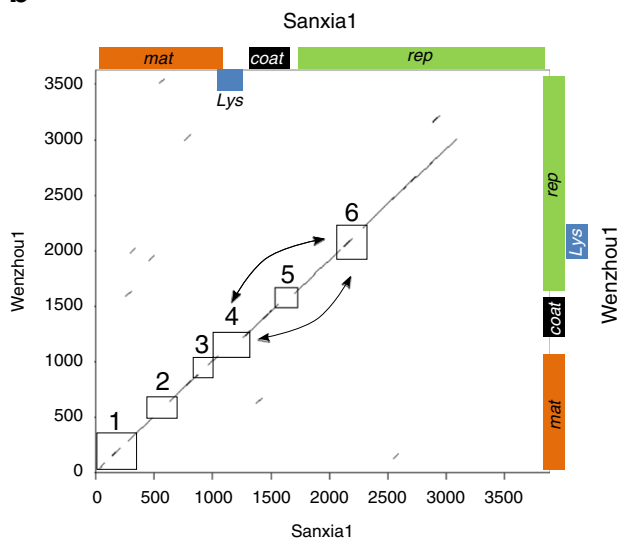

d

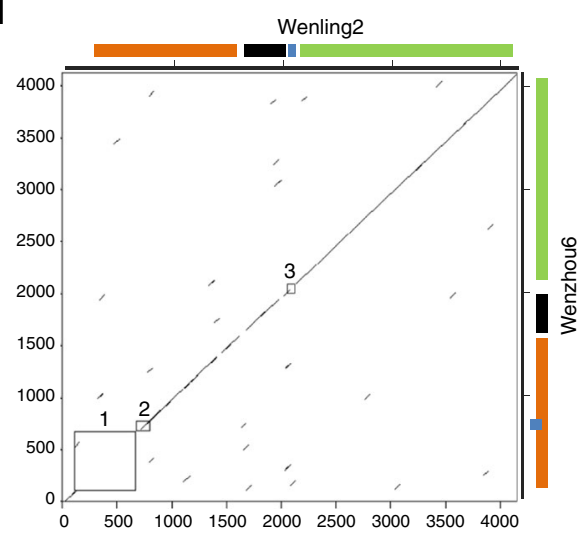

e

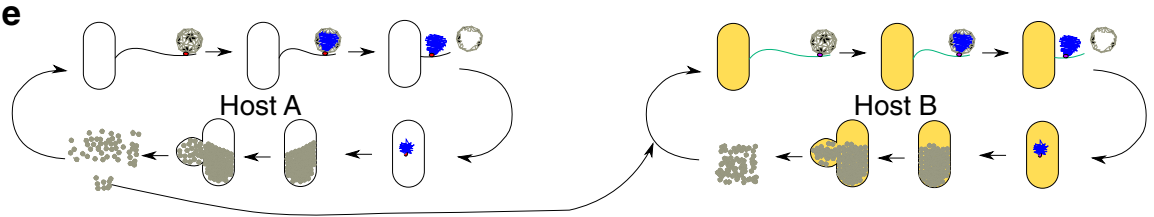

$\mathbf{f}$

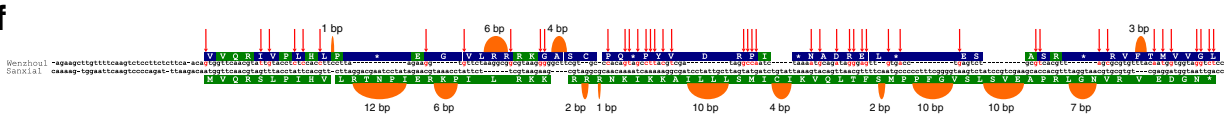

Fig. 6 De novo gene evolution in ssRNA phages sourced from relatively close geographic locations. a Geographic locations and the invertebrate animal sources for the transcriptomes that contained the genomes of Wenzhou1, Wenzhou6, Wengling2, and Sanxia1. Silhouette images were obtained from phylopic. org, courtesy of Christoph Schomburg (shrimp), Joanna Wolfe and T. Michael Keesey (pan-Crustacea), and Scott Hartman (snail) under CCO 1.0 (https:// creativecommons.org/publicdomain/zero/1.0/). b Nucleotide dot plot between Sanxia1 and Wenzhou1 genomes. The gaps in the first $\sim 3000$ bases of the alignment are boxed and numbered. Bi-directional arrows indicate the shift in the location of the sgls. $\mathbf{c}$ Dot plot of the maturation protein primary structures of Sanxia1 and Wenzhou1. d Similar to $\mathbf{b}$ but Wenzhou6 vs Wenling2 comparison. e Model for de novo lysis gene evolution after changing pili specificity and presumably the host. $\mathbf{f}$ Sequence alignment of the region represented in box 4 of panel $\mathbf{b}$ with base changes highlighted red and indicated with red arrows. The indels are shown as orange loops with the size of the indel indicated above the loops. The translated reading frames are shown above (Wenzhou1) or below (Sanxia1) the nucleotide sequence and the amino acids shared between $\left.\mathrm{Sg}\right|^{S a n x i a 1}$ and Wenzhou1 reading frame are colored green.

Gyrotory G76) until the O.D $D_{550}$ reached 0.2 , at which point the cultures were induced with arabinose $(0.4 \% \mathrm{w} / \mathrm{v}$ final concentration). Two hours post induction the cultures were harvested and centrifuged for 10 minutes at $10,000 \times g$ at $4{ }^{\circ} \mathrm{C}$. The supernatant was filtered through $0.22 \mu \mathrm{m}$ syringe filter into a new $50 \mathrm{~mL}$ falcon tube. At this point $1 / 10$ volume of $3 \mathrm{M}$ Sodium acetate $\mathrm{pH} 5.2$ and 1 volume of isopropanol was added to the filtrate, and then passed through a DNA spin column $\left(\right.$ Econospin $\left.^{\mathrm{TM}}\right)$. The columns were then washed with $2 \mathrm{~mL}$ of Qiagen PE wash buffer and bound DNA was eluted in $50 \mu \mathrm{L}$ of sterile water. Then $5 \mu \mathrm{L}$ of the eluted DNA was transformed back into DH5 $\alpha \mathrm{T}^{\mathrm{R}}$ cells and transformants were pooled and subjected to another round of plasmid release as described above. The whole process was repeated for a total of two to three rounds.

Dot plots and multiple interrelated sequence doT (MIST) plots. Nucleotide dot plots were generated with dotmatcher ${ }^{33}$ with window size and threshold set at 40.0 and 50.0, respectively. MIST v3 was used to generate complete $\mathrm{NxN}$ plot of related genomes. The code for dotmatcher and MIST tools are available from CPT Galaxy Tools, which is available at https://doi.org/10.5281/zenodo.4048782.

Multiple sequence alignments (MSA), pair-wise alignments, and phylogenetic analyses. The MSA, phylogenetic analyses of Mat and Rep primary structures, and pair-wise alignments of Sgls were done using CLC Genomics Workbench 8.0.1 (Qiagen). The MSAs were done with gap open and extension costs of 10.0 and 1.0, respectively. Circular cladograms were constructed using Neighbor-Joining method and bootstrap analyses with 1000 replicates.

Codon usage (CU) analysis. CU was determined using Measure Independent of Length and Composition (MILC) method ${ }^{34}$. The $\mathrm{CU}$ analyses were done using an 
R package, coRdon, from Bioconductor ${ }^{35}$. A R-script was written to calculate and plot MILC values for individual mat and rep genes (Supplementary Software). The codon distribution was calculated from corresponding mat and rep genes and plotted on $X$ and $Y$ axis, respectively.

Q $\beta$ replicase structural rendering. The relative locations of the functional-sgls were rendered on the crystal structure of $\mathrm{Q} \beta$ replicase beta subunit (PDB: 4R71) using the UCSF Chimera package ${ }^{36}$.

Reporting summary. Further information on research design is available in the Nature Research Reporting Summary linked to this article.

\section{Data availability}

All data generated and analyzed during the current study are available from the corresponding author upon reasonable request. The source data for Figs. 4a, b, d, 5d, e, and Supplementary Fig. 3a-d are available in the Source data file. The ssRNA phage genome sequences used in this study were sourced from previously deposited and/or published sources ${ }^{18,19}$. Of the 244 genomes, 96 have GenBank accession numbers (see Supplementary Data 1) and the rest are available at https://doi.org/10.1371/journal. pbio.1002409.s001. The structure of $Q \beta$ replicase beta subunit was obtained from Protein Data Bank (PDB:4R71). Source data are provided with this paper.

\section{Code availability}

The code used in this study for MIST and dot plots is available from public repository at https://doi.org/10.5281/zenodo.4048782. The R scripts used for codon usage analysis and for generating Fig. 6a are provided as Supplementary Software.

Received: 21 August 2020; Accepted: 30 October 2020;

Published online: 26 November 2020

\section{References}

1. Gorzelnik, K. V. et al. Asymmetric cryo-EM structure of the canonical allolevivirus $\mathrm{Q} \beta$ reveals a single maturation protein and the genomic ssRNA in situ. Proc. Natl Acad. Sci. USA 113, 11519-11524 (2016).

2. Cui, Z. et al. Structures of $\mathrm{Q} \beta$ virions, virus-like particles, and the $\mathrm{Q} \beta-\mathrm{Mur} A$ complex reveal internal coat proteins and the mechanism of host lysis. Proc. Natl Acad. Sci. USA 114, 11697-11702 (2017).

3. Dai, X. et al. In situ structures of the genome and genome-delivery apparatus in a single-stranded RNA virus. Nature 541, 112-116 (2017).

4. Dent, K. C. et al. The asymmetric structure of an icosahedral virus bound to its receptor suggests a mechanism for genome release. Structure 21, 1225-1234 (2013).

5. Meng, R. et al. Structural basis for the adsorption of a single-stranded RNA bacteriophage. Nat. Commun. 10, 3130 (2019).

6. Beremand, M. N. \& Blumenthal, T. Overlapping genes in RNA phage: a new protein implicated in lysis. Cell 18, 257-266 (1979).

7. Winter, R. B. \& Gold, L. Overproduction of bacteriophage $\mathrm{Q} \beta$ maturation $\left(\mathrm{A}_{2}\right)$ protein leads to cell lysis. Cell 33, 877-885 (1983).

8. Bernhardt, T. G., Wang, I. N., Struck, D. K. \& Young, R. A protein antibiotic in the phage $\mathrm{Q} \beta$ virion: diversity in lysis targets. Science 292, 2326-2329 (2001).

9. Holtje, J. V. \& van Duin, J. In Microbial Cell Wall Synthesis and Autolysis (ed. Nombela, C.) 195-199 (Elsevier Science Publishers, 1984).

10. Chamakura, K. R., Tran, J. S. \& Young, R. MS2 lysis of Escherichia coli depends on host chaperone DnaJ. J. Bacteriol. 199, e00058-00017 (2017).

11. Kannoly, S., Shao, Y. \& Wang, I. N. Rethinking the evolution of singlestranded RNA (ssRNA) bacteriophages based on genomic sequences and characterizations of two R-plasmid-dependent ssRNA phages, C-1 and Hgall. J. Bacteriol. 194, 5073-5079 (2012).

12. Rumnieks, J. \& Tars, K. Diversity of pili-specific bacteriophages: genome sequence of IncM plasmid-dependent RNA phage M. BMC Microbiol. 12, 277 (2012).

13. Kazaks, A., Voronkova, T., Rumnieks, J., Dishlers, A. \& Tars, K. Genome structure of Caulobacter phage phiCb5. J. Virol. 85, 4628-463 (2011).

14. Klovins, J., Overbeek, G. P., van den Worm, S. H., Ackermann, H. W. \& van Duin, J. Nucleotide sequence of a ssRNA phage from Acinetobacter: kinship to coliphages. J. Gen. Virol. 83, 1523-1533 (2002)

15. Pourcel, C., Midoux, C., Vergnaud, G. \& Latino, L. A carrier state is established in Pseudomonas aeruginosa by phage LeviOr01, a newly isolated ssRNA levivirus. J. Gen. Virol. 98, 2181-2189 (2017).
16. Chamakura, K. R. et al. A viral protein antibiotic inhibits lipid II flippase activity. Nat. Microbiol. 2, 1480-1484 (2017).

17. Chamakura, K. R., Edwards, G. B. \& Young, R. Mutational analysis of the MS2 lysis protein L. Microbiology 163, 961-969 (2017).

18. Krishnamurthy, S. R., Janowski, A. B., Zhao, G., Barouch, D. \& Wang, D. Hyperexpansion of RNA Bacteriophage Diversity. PLoS Biol. 14, e1002409 (2016).

19. Shi, M. et al. Redefining the invertebrate RNA virosphere. Nature 540, 539-543 (2016).

20. Starr, E. P., Nuccio, E. E., Pett-Ridge, J., Banfield, J. F. \& Firestone, M. K. Metatranscriptomic reconstruction reveals RNA viruses with the potential to shape carbon cycling in soil. Proc. Natl Acad. Sci. USA 116, 25900-25908 (2019).

21. Callanan, J. et al. Expansion of known ssRNA phage genomes: from tens to over a thousand. Sci. Adv. 6, eaay5981 (2020).

22. Venkataraman, S., Prasad, B. \& Selvarajan, R. RNA dependent RNA polymerases: insights from structure, function and evolution. Viruses 10,76 (2018).

23. Kidmose, R. T., Vasiliev, N. N., Chetverin, A. B., Andersen, G. R. \& Knudsen, C. R. Structure of the Qbeta replicase, an RNA-dependent RNA polymerase consisting of viral and host proteins. Proc. Natl Acad. Sci. USA 107, 10884-10889 (2010)

24. Hatfull, G. F. Microbiology. The great escape. Science 292, 2263-2264 (2001)

25. Chamakura, K. \& Young, R. Phage single-gene lysis: finding the weak spot in the bacterial cell wall. J. Biol. Chem. 294, 3350-3358 (2019).

26. Fiers, W. et al. Complete nucleotide sequence of bacteriophage MS2 RNA primary and secondary structure of the replicase gene. Nature 260, 500-507 (1976).

27. Sanger, F. et al. Nucleotide sequence of bacteriophage $\phi X 174$ DNA. J. Mol. Biol. 125, 225-246 (1978)

28. Wahba, A. J. et al. Subunit I of Qbeta replicase and $30 \mathrm{~S}$ ribosomal protein S1 of Escherichia coli. Evidence for the identity of the two proteins. J. Biol. Chem. 249, 3314-3316 (1974).

29. Kamen, R. Characterization of the subunits of Q-beta replicase. Nature $\mathbf{2 2 8}$ 527-533 (1970).

30. Blumenthal, T., Landers, T. A. \& Weber, K. Bacteriophage $\mathrm{Q} \beta$ replicase contains the protein biosynthesis elongation factors EF Tu and EF Ts. Proc. Natl Acad. Sci. USA 69, 1313-1317 (1972).

31. Carmichael, G. G., Weber, K., Niveleau, A. \& Wahba, A. J. The host factor required for RNA phage Qbeta RNA replication in vitro. Intracellular location, quantitation, and purification by polyadenylate-cellulose chromatography. $J$. Biol. Chem. 250, 3607-3612 (1975).

32. Dolja, V. V. \& Koonin, E. V. Metagenomics reshapes the concepts of RNA virus evolution by revealing extensive horizontal virus transfer. Virus Res. 244, 36-52 (2018).

33. Krumsiek, J., Arnold, R. \& Rattei, T. Gepard: a rapid and sensitive tool for creating dotplots on genome scale. Bioinformatics 23, 1026-1028 (2007).

34. Supek, F. \& Vlahovicek, K. Comparison of codon usage measures and their applicability in prediction of microbial gene expressivity. BMC Bioinforma. $\mathbf{6}$, 182 (2005).

35. coRdon: Codon usage analysis and prediction of gene expressivity. v. R package version 1.7.0 (Bioconductor, 2020).

36. Pettersen, E. F. et al. UCSF Chimera-a visualization system for exploratory research and analysis. J. Comput. Chem. 25, 1605-1612 (2004).

\section{Acknowledgements}

This work was supported by Public Health Service Grant GM27099 and R35GM136396 to R.Y. and by the Center for Phage Technology at Texas A\&M University, jointly sponsored by Texas A\&M AgriLife. Additional funding for this project and support of J.S. Tran was provided by the Beckman Scholars Program administered by the Arnold and Mabel Beckman Foundation. The authors thank Curtis Ross and Anthony Criscione for their assistance with the MIST plot.

\section{Author contributions}

K.C. and R.Y. designed the study and the analyzed results. K.C., J.T., C.O., H.L., K.G., E.T., and L.M. annotated, cloned, and tested sgls. K.G., H.L., and C.O. performed lysis profiles of functional Sgls. S.A. constructed mutant library and performed plasmid release of beihai9_2. H.L. constructed mutant libraries of sgls of Hubei7 and Hubei8 and performed plasmid release and assisted in making the figures. K.C. and R.Y. prepared and edited the manuscript

\section{Competing interests}

The authors declare no competing interests. 


\section{Additional information}

Supplementary information is available for this paper at https://doi.org/10.1038/s41467020-19860-0.

Correspondence and requests for materials should be addressed to R.Y.

Peer review information Nature Communications thanks the anonymous reviewers for their contribution to the peer review of this work.

Reprints and permission information is available at http://www.nature.com/reprints

Publisher's note Springer Nature remains neutral with regard to jurisdictional claims in published maps and institutional affiliations. (c) (i) Open Access This article is licensed under a Creative Commons Attribution 4.0 International License, which permits use, sharing, adaptation, distribution and reproduction in any medium or format, as long as you give appropriate credit to the original author(s) and the source, provide a link to the Creative Commons license, and indicate if changes were made. The images or other third party material in this article are included in the article's Creative Commons license, unless indicated otherwise in a credit line to the material. If material is not included in the article's Creative Commons license and your intended use is not permitted by statutory regulation or exceeds the permitted use, you will need to obtain permission directly from the copyright holder. To view a copy of this license, visit http://creativecommons.org/licenses/by/4.0/.

(C) The Author(s) 2020 